\title{
SOME INTEGRAL INEQUALITIES OF TWO GEOMETRIC INVARIANTS
}

\author{
BY BANG-YEN CHEN 1
}

Communicated by Shlomo Sternberg, August 12, 1974

Let $M$ be an $n$-dimensional manifold immersed in a euclidean $m$-space $E^{m}$. Let $S$ and $\alpha$ be the length of second fundamental form and the length of mean curvature vector and let $\rho$ be the scalar curvature of $M$. Then $\rho=$ $n^{2} \alpha^{2}-S^{2}$. From Proposition 2.2 of [2], $\rho$ satisfies the following pinching property:

$$
-S^{2} \leqslant \rho \leqslant(n-1) S^{2} .
$$

Let $F$ be a field and let $H_{i}(M ; F)$ be the $i$ th homology group of $M$ over $F$. We define a topological invariant $\beta(M)$ by

$$
\beta(M)=\max \left\{\sum_{i=0}^{n} \operatorname{dim} H_{i}(M ; F): F \text { fields }\right\} .
$$

The purpose of this note is to announce the following results. The detailed proofs will appear elsewhere.

THEOREM 1. Let $M$ be an n-dimensional closed manifold immersed in a euclidean $m$-space $E^{m}$. Then

$$
\int_{M} S^{n} d V \geqslant \gamma \beta(M)
$$

where

$$
\gamma= \begin{cases}n^{n / 2} c_{n} / 2, & \text { if } n \text { is even } \\ n^{(n+1) / 2} c_{n+1} c_{m-1} / 2 c_{m}(m-1)^{1 / 2}, & \text { if } n \text { is odd }\end{cases}
$$

and $c_{n}$ is the area of unit n-spheres. The equality sign holds only when $M$ is imbedded as a hypersphere of a linear $(n+1)$-subspace of $E^{m}$.

AMS (MOS) subject classifications (1970). Primary 53B25, 53C40.

Key words and phrases. Length of second fundamental form, total mean curvature, scalar curvature, homology groups.

${ }^{1}$ This work was partially supported by NSF grant GP-36684. 
This theorem was proved by using several lemmas given in [1, Chapter VII]. By applying Theorem 1 we have the following theorem on total mean curvature.

THEOREM 2. Let $M$ be an n-dimensional closed manifold immersed in $E^{m}$. If there is a $\delta>-1$ such that $\delta S^{2} \leqslant \rho \leqslant(n-1) S^{2}$, then

$$
\int_{M} \alpha^{n} d V \geqslant(1+\delta)^{n / 2} \gamma \beta(M) / n^{n} \text {. }
$$

The equality sign of (3) holds only when $\delta=n-1$ and $M$ is imbedded as a hypersphere in an $(n+1)$-dimensional linear subspace of $E^{m}$.

Some special cases of these results were obtained in [2].

\section{BIBLIOGRAPHY}

1. B.-Y. Chen, Geometry of submanifolds, Dekker, New York, 1973.

2. - On the total curvature of immersed manifolds. II. Mean curvature and length of second fundamental form, Amer. J. Math. 94 (1972), 799-809. MR 47 \#7660.

DEPARTMENT OF MATHEMATICS, MICHIGAN STATE UNIVERSITY, EAST LANSING, MICHIGAN 48824 